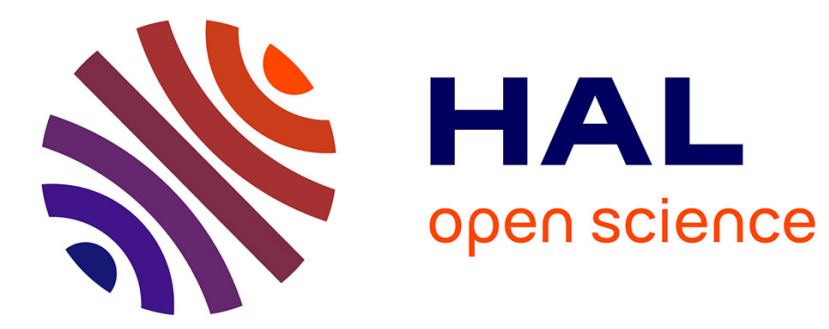

\title{
Streamwise forced oscillations of circular and square cylinders
}

Daniel Tudball-Smith, Justin S. Leontini, John Sheridan, David Lo Jacono

\section{To cite this version:}

Daniel Tudball-Smith, Justin S. Leontini, John Sheridan, David Lo Jacono. Streamwise forced oscillations of circular and square cylinders. Physics of Fluids, 2012, 10.1063/1.4758286 . hal-00752232

\section{HAL Id: hal-00752232 \\ https://hal.science/hal-00752232}

Submitted on 15 Nov 2012

HAL is a multi-disciplinary open access archive for the deposit and dissemination of scientific research documents, whether they are published or not. The documents may come from teaching and research institutions in France or abroad, or from public or private research centers.
L'archive ouverte pluridisciplinaire HAL, est destinée au dépôt et à la diffusion de documents scientifiques de niveau recherche, publiés ou non, émanant des établissements d'enseignement et de recherche français ou étrangers, des laboratoires publics ou privés. 


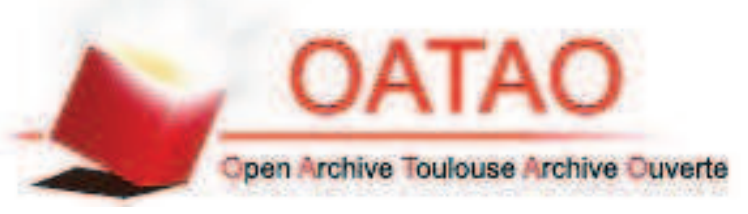

\section{Open Archive TOULOUSE Archive Ouverte (OATAO)}

OATAO is an open access repository that collects the work of Toulouse researchers and makes it freely available over the web where possible.

This is an author-deposited version published in : http://oatao.univ-toulouse.fr/ Eprints ID : 6866

To link to this article : DOI: $10.1063 / 1.4758286$

URL : http://dx.doi.org/10.1063/1.4758286

To cite this version : Tudball-Smith, Daniel and Leontini, Justin S. and Sheridan, John and Lo Jacono, David Streamwise forced oscillations of circular and square cylinders. (2012) Physics of Fluids ( $\left.{ }^{\circ} 111703\right)$. ISSN 1070-6631

Any correspondence concerning this service should be sent to the repository administrator: staff-oatao@listes.diff.inp-toulouse.fr 


\title{
Streamwise forced oscillations of circular and square cylinders
}

\author{
Daniel Tudball-Smith, ${ }^{1}$ Justin S. Leontini, ${ }^{1, a)}$ John Sheridan, ${ }^{1}$ \\ and David Lo Jacono $0^{1,2,3}$ \\ ${ }^{1}$ Fluids Laboratory for Aeronautical and Industrial Research (FLAIR), \\ Department of Mechanical and Aerospace Engineering, Monash University, \\ Melbourne, Victoria 3800, Australia \\ ${ }^{2}$ Université de Toulouse, INPT, UPS, IMFT, Allée Camille Soula, F-31400 Toulouse, France \\ ${ }^{3}$ CNRS, IMFT, F-31400 Toulouse, France
}

\begin{abstract}
The modification of a cylinder wake by streamwise oscillation of the cylinder at the vortex shedding frequency of the unperturbed cylinder is reported. Recent numerical simulations [J. S. Leontini, D. Lo Jacono, and M. C. Thompson, "A numerical study of an inline oscillating cylinder in a free stream," J. Fluid Mech. 688, 551-568 (2011)] showed that this forcing results in the primary frequency decreasing proportionally to the square of the forcing amplitude, before locking to a subharmonic at higher amplitudes. The experimental results presented here show that this behavior continues at higher Reynolds numbers, although the flow is three-dimensional. In addition, it is shown that this behavior persists when the body is a square cross section, and when the frequency of forcing is detuned from the unperturbed cylinder shedding frequency. The similarity of the results across Reynolds number, geometry, and frequency suggests that the physical mechanism is applicable to periodic forcing of the classic von Kármán vortex street, regardless of the details of the body which forms the street.
\end{abstract}

This study reports on an experimental study of cylinders undergoing forced streamwise oscillations. Streamwise forcing is just one method of active open-loop flow control that can be employed on the cylinder wake. Others include, but are not limited to, transverse (cross-stream) oscillation, ${ }^{1-4}$ rotational oscillation of the cylinder, ${ }^{5-8}$ constant rotation of the cylinder, ${ }^{9,10}$ and actuation by synthetic jets from the surface of the cylinder and base bleed. ${ }^{11-13}$

Previous studies of streamwise forced oscillation ${ }^{14-17}$ have investigated the synchronization between the forcing and the vortex shedding. These studies report that the vortex shedding locks to a subharmonic of the forcing (vortex shedding at half the frequency of the forcing), particularly for forcing frequencies around twice $f_{\mathrm{so}}$, the shedding frequency from the unperturbed cylinder. Barbi et al..$^{15}$ and Ongoren and Rockwell ${ }^{18}$ also report that a synchronized symmetric wake structure, different from the classic von Kármán street, can be achieved depending on the amplitude and frequency of forcing, typically being found for high values of the frequency. Yokoi and Kamemoto ${ }^{19}$ found a series of synchronized states, depending on forcing frequency, amplitude, and Reynolds number. These states could be synchronized to either the forcing frequency or to its first subharmonic, particularly when the forcing frequency was close to $f_{\text {so }}$, a finding confirmed and extended by Cetiner and Rockwell. ${ }^{20}$

Perdikaris, Kaitsis, and Triantafyllou ${ }^{21}$ numerically investigated a cylinder oscillating at the unperturbed shedding frequency, $f_{\text {so }}$, for a Reynolds number $R e=400$. This study found that, for low amplitudes, the flow remained in a state similar to that of an unperturbed cylinder. At high values of amplitude, they found locking to the first subharmonic of the forcing. At moderate amplitudes, they

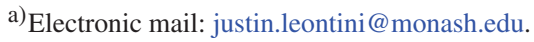


(a)

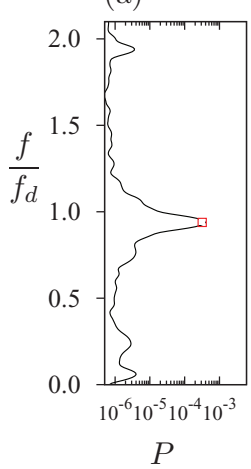

(b)

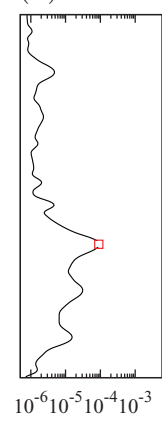

(c)

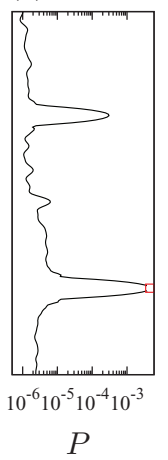

(a)

(b)

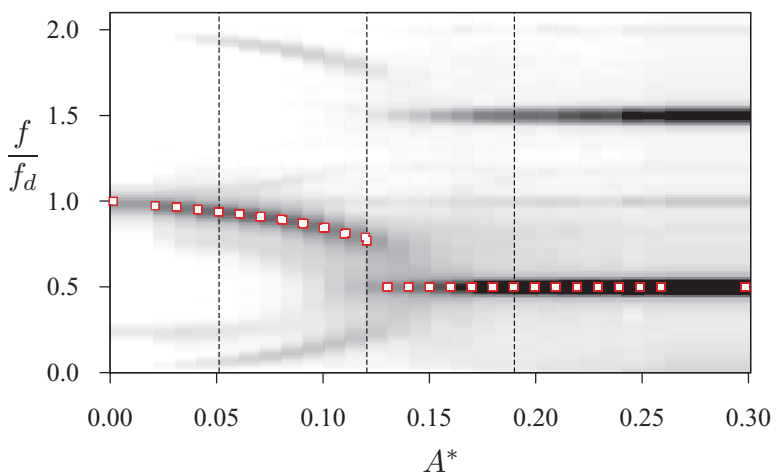

FIG. 1. Power spectral density of the lift force on the circular cylinder at $R e=5000$ as a function of $A^{*}$. Line plots labeled (a), (b), and (c) are taken at $A^{*}=0.05,0.12$, and 0.19 , respectively, and are indicated by the dashed lines on the power spectral density isocontour. The square points represent the primary frequency, $f_{\mathrm{s}}$ for each $A^{*}$.

reported chaos, with disorganized vortex shedding. This chaos was attributed to mode competition between the reflection-symmetric forcing, and the spatio-temporally symmetric Kármán street.

A recent numerical study ${ }^{22}$ at $R e=175$ using fine increments of amplitude and forcing at $f_{\text {so }}$ found that, for low to moderate amplitudes, the primary frequency of vortex shedding, $f_{\mathrm{s}}$, decreased with increasing non-dimensional amplitude $A^{*}=A / D$, where $A$ is the amplitude of forcing and $D$ is the cylinder diameter, scaling as $f_{\mathrm{s}} \propto A^{* 2}$ as suggested in Perdikaris, Kaitsis, and Triantafyllou. ${ }^{21}$ The dynamics were shown to be dictated by the interaction of this new primary frequency $f_{\mathrm{s}}$ and the forcing frequency, typically resulting in a quasi-periodic, amplitude and frequency modulated flow. However, nonlinear synchronization was found when the primary frequency and driving frequency made an integer ratio, and a series of subharmonic modes was discovered from the $1 / 8$ th mode at low amplitudes all the way to the first $1 / 2$ subharmonic.

The current study has two primary aims. The first is to investigate experimentally whether the dynamics reported in the previous numerical studies ${ }^{21,22}$ persist at higher Reynolds numbers. The second is to investigate whether the dynamics are similar for differently shaped bodies. Also presented are results from experiments where the forcing frequency is detuned from $f_{\text {so }}$.

Experiments were conducted using the Fluids Laboratory for Aeronautical and Industrial Research (FLAIR) water channel at Monash University. This facility is a free surface water channel with $800 \mathrm{~mm}$ depth, $600 \mathrm{~mm}$ width, and $4000 \mathrm{~mm}$ length with a test section of $500 \mathrm{~mm}$ width and $650 \mathrm{~mm}$ depth. This facility has been previously used for similar bluff-body wake studies. ${ }^{23,24}$

Square/circular cylinders of $25 \mathrm{~mm}$ width/diameter were oscillated using a high resolution stepper motor connected to a traverse, which allowed for linear translational motion. ${ }^{25}$ Position was confirmed using a hollow bore optical encoder attached to the shaft of the stepper. Lift and drag forces on the cylinders were measured using strain gauges on a load cell positioned above the free surface at a sampling frequency of $100 \mathrm{~Hz}$. Both square and circular cylinders were attached to the load cell such that they passed through the test section with the end of the cylinder finishing $<10 \mathrm{~mm}$ from the bottom of the channel. Reynolds number was defined using the freestream velocity and the cross-stream width of the body. The range of Reynolds number examined was $R e \in[1500-5500]$ and [3750-6300] for the square and circular cylinders, respectively.

The shedding frequency $f_{\text {so }}$ was determined from a stationary body using frequency analysis of the lift force.

The frequency content of the lift force was determined using a method based on a fast Fourier transform (FFT). The total time signal was broken into a series of segments, which overlapped by half of the length of the segment. An FFT was taken of each segment, and the results of all segments were then averaged. This process essentially adds a type of high-pass filtering, removing the influence of very low frequencies. Spectra produced using this method are shown on the left-hand side of Figure 1. 

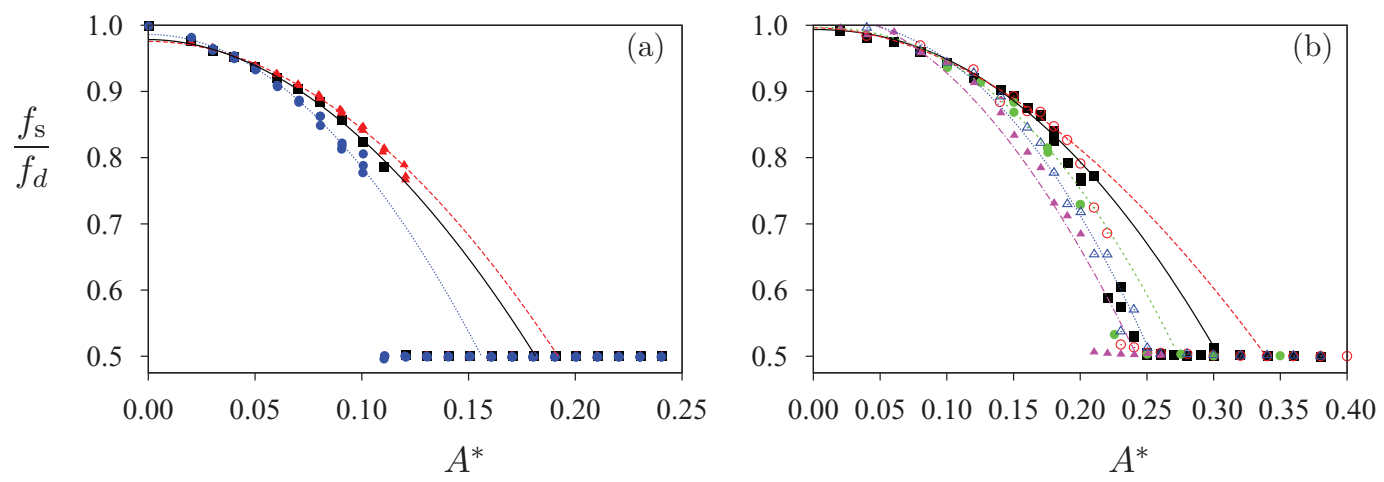

FIG. 2. Primary shedding frequency, $f_{\mathrm{s}}$, normalized by the driving frequency $f_{\mathrm{d}}$, as a function of $A^{*}$ for (a) the circular cylinder and (b) the square cross section, at various Re. Points are measured frequency, lines are a power-law fit of the form $f_{\mathrm{s}} / f_{\mathrm{d}}=c-a A^{* b}$ (see Table I and II for the coefficient values). For (a): $R e=3750(\bullet), R e=5000(\boldsymbol{\Delta}) R e=6300(\boldsymbol{\square})$. For (b): $\operatorname{Re}=1500(\Delta) ; \operatorname{Re}=2500(\triangle) ; \operatorname{Re}=3550(\bullet) ; \operatorname{Re}=4500(\bigcirc) ; \operatorname{Re}=5500(\mathbf{\square})$. For all values of $R e, f_{\mathrm{s}}$ initially decreases with $A^{* 2}$ and synchronizes to a period doubled state at higher amplitudes. However, the transition between these two states is more abrupt for the circular cylinder case. Note that the symbols for each $R e$ in this figure overlap when $f_{\mathrm{s}} / f_{d}=0.5$.

Figure 1 shows the development of the frequency content for a circular cylinder, at $R e=5000$, with increasing amplitude of oscillation. The three example spectra on the left are for $A^{*}=0.05, A^{*}$ $=0.12$, and $A^{*}=0.20$, respectively. The frequencies have been normalized by the driving frequency $f_{d}=f_{\text {so }}$. These images show that as the amplitude is increased, the primary frequency of response $f_{\mathrm{s}}$ (marked with the square point) is lowered away from $f_{\text {so }}$, before reaching $f_{d} / 2$. The map on the right-hand side of Figure 1 shows this trend very clearly. The contours on this image represent the energy for a given frequency component as a function of $A^{*}$. This figure was produced by essentially "stacking" the spectra for each value of $A$ * next to each other. These contours show that the frequency content of the flow response is a smooth function of the amplitude, up to $A^{*} \simeq 0.12$. For $A^{*}<0.12$, $f_{\mathrm{s}}$ (again highlighted with the square points) decreases apparently quadratically. The contours also show that over this range of $A^{*}$, two other frequencies contain small, but non-negligible energy; one at $f_{d}-f_{\mathrm{s}}$, and another at $f_{d}+f_{\mathrm{s}}$. At $A^{*} \simeq 0.12$, there is a sudden drop in the primary frequency to $f_{d} / 2$, and the flow response remains locked to this subharmonic of the driving frequency with further increases in amplitude. This behavior is in agreement with the numerical work of Leontini, Lo Jacono, and Thompson ${ }^{22}$ despite an order of magnitude difference in Re. This observation is not necessarily expected, given the high level of three-dimensionality in the experiments.

The aforementioned numerical work reported that the initial decrease in $f_{\mathrm{s}}$ is of the form $f_{\mathrm{s}} / f_{\mathrm{d}}$ $=1-a A^{* 2}$. Figure 2(a) shows $f_{\mathrm{s}}$ as a function of $A^{*}$ for three values of $R e$. The data for $A^{*}$ lower than that at which the flow locks to the subharmonic of the driving frequency have been fitted with a power-law curve of the form $f_{\mathrm{s}} / f_{\mathrm{d}}=c-a A^{* b}$ in a least-squares sense allowing all 3 constants $(a$, $b$, and $c$ ) to vary.

Letting the constant $c$ "float" accounted for any experimental error in determining $f_{\mathrm{d}}$ from the unperturbed cylinder experiments. In all cases, the value of $c$ was seen to vary slightly but remained close to unity. The curves corresponding to these fits are plotted in Figure 2(a), and the coefficients are shown in Table I.

TABLE I. Values of the coefficients in the fit $f_{\mathrm{s}} / f_{\mathrm{d}}=c-a A^{* b}$, for $A^{*}<0.1$ for the circular cylinder. For the range of $R e$ tested, the exponent $b$ remains close to 2 . Note that the constant parameter $c$ is close to unity.

\begin{tabular}{lccr}
\hline \hline$R e$ & $a$ & $b$ & $c$ \\
\hline 3750 & 19.52 & 1.99 & 0.98 \\
5000 & 12.19 & 1.96 & 0.97 \\
6300 & 13.13 & 1.94 & 0.98 \\
\hline \hline
\end{tabular}


(a)

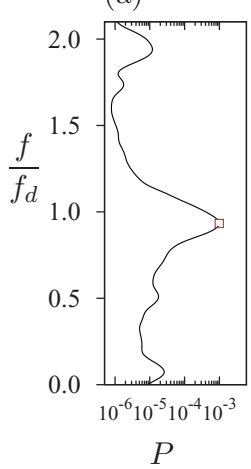

(b)

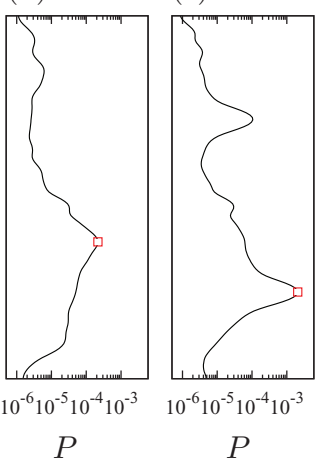

(a)

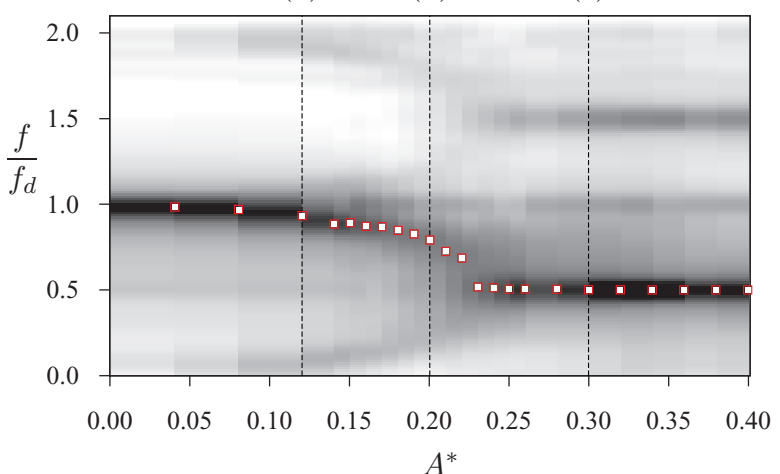

FIG. 3. Power spectral density of the lift force on the square cylinder at $R e=4500$ as a function of $A^{*}$. Line plots labeled (a), (b), and (c) are taken at $A^{*}=0.11,0.20$, and 0.30 , respectively, and are indicated by the dashed lines on the power spectral density isocontour. The dotted symbols represent the maximum frequency, $f_{\mathrm{s}}$ for each $A^{*}$.

Table I shows that for all the values of $R e$, the exponent $b$ remains close to 2 . The small variation in $b$ indicates that even at these higher values of $R e$, the primary frequency for the circular cylinder decreases with $A^{* 2}$.

To further test the generality of the findings, a similar approach was followed using a square cylinder. Figure 3 shows examples of spectra for the square cylinder at $R e=4500$; similar to Figure 1, three example spectra are shown on the left-hand side, while the image on the right shows contours of energy as a function of frequency of response and amplitude, produced by stacking the spectra for each $A^{*}$ next to each other. The square points on the figure mark the primary frequency of response for each value of $A^{*}$. Similar to the circular cylinder, this figure shows that for a square cylinder, the strongest frequency decreases from $f \approx f_{\text {so }}$ as the amplitude of oscillation is increased, before locking to $f=f_{\mathrm{so}} / 2$. Again, energy at the secondary frequencies of $f_{\mathrm{d}}-f_{\mathrm{s}}$ and $f_{\mathrm{d}}+f_{\mathrm{s}}$ is evident in the contours.

Figure 2(b) presents the primary frequency of shedding $f_{\mathrm{s}}$ as a function of $A^{*}$, for a series of values of $R e$. The square cylinder follows a similar trend to the circular cylinder. At low amplitudes $f_{\mathrm{s}}$ decreases from the driving frequency with increasing $A^{*}$, and transitions to a period doubled locked-in state at higher amplitudes. Though some differences are observed in the transition to this locked subharmonic state, the results suggest that this behavior is generic for spatio-temporally symmetric vortex street wakes, regardless of the details of the body, which formed them. However, wakes which do not possess this symmetry (such as those generated from non-symmetric bodies), or which have different vortex configurations (such as those that could be produced by more complex forcing functions) could behave in a different manner.

The trend of $f_{\mathrm{s}}$ decreasing with $A^{* 2}$, found for the circular cylinder, is recovered from the square cylinder data, at least for $A^{*}<0.15$. As for the circular cylinder, making a power-law fit to $f_{\mathrm{s}}$ as a function of $A^{*}$ results in exponents close to 2 . The exponents of these fits, for a range of values of $R e$, are presented in Table II.

TABLE II. Values of the coefficients in the fit $f_{\mathrm{s}} / f_{\mathrm{d}}=c-a A^{* b}$, for $A^{*}<0.2$ for the square cylinder. For the range of $R e$ tested, the exponent $b$ remains close to 2 . Note that the constant parameter $c$ is close to unity.

\begin{tabular}{lccr}
\hline \hline$R e$ & $a$ & $b$ & $c$ \\
\hline 1500 & 8.05 & 1.93 & 1.02 \\
2500 & 11.4 & 2.26 & 1.01 \\
3550 & 9.26 & 2.25 & 0.99 \\
4500 & 3.78 & 1.88 & 0.99 \\
5500 & 6.40 & 2.15 & 0.99 \\
\hline \hline
\end{tabular}


Close inspection of the data shows some impact of the body geometry. First, for the square cylinder the locking to a period doubled state occurs at a higher non-dimensional amplitude $\left(A^{*}\right)$ compared to the circular cylinder. Second, for the circular cylinder, the transition to this period doubled region occurs abruptly. However, for the square cylinder, the frequency initially follows the trend of $A^{* 2}$ until around $A^{*}=0.15$, and then rapidly, but smoothly, decreases to $f_{\mathrm{d}} / 2$ over the range $0.15 \leq A^{*} \leq 0.2$. This transitional region appears to have increased disorder with a broader spectrum response than the other regions. The spectrum shown in Figure 3(b), for $A^{*}=0.20$ when $R e=4500$ shows this. Although it is still possible to find a peak frequency in the signal, it is not as distinct or dominant. A broad spectrum response is observed at amplitudes in the transition range, with no significantly dominant peak. It is possible that this broadband response is due to a competition between modes, one with vortex shedding at a frequency dictated by $A^{* 2}$, the other at the subharmonic frequency of the driving, $f_{\mathrm{d}} / 2$. However, this mode competition does not simply lead to periods of time oscillating in one mode, then periods in the other mode. Rather, the flow oscillations are quite irregular, and so a definitive conclusion on the cause of this chaos has not been found.

The circular cylinder data of Figure 1 and the square cylinder data of Figure 3 show that there is some dependence on $R e$, in terms of the amplitude at which locking to the period doubled state occurs, and the steepness of the initial variation of $f_{\mathrm{s}}$ with $A^{*}$ (this is also reflected in the variation of $a$ in Table II). This variation appears to be rather complex, as the amplitude at the period doubled locking varies non-monotonically. This perhaps is not surprising, considering that there are a number of subtle changes in the wake and shear layer formation that occur in the range of Re tested, even for a stationary cylinder. ${ }^{26,27}$ This complicated dependence on $R e$ highlights the underlying complexity of even the canonical flow of the cylinder wake, and the difficulties faced when trying to devise any control method, including periodic forcing. The studies of Parezanović and Cadot, ${ }^{28,29}$ using a passive control method of a small cylinder positioned close to the main cylinder, also highlighted this complexity, showing that the global frequency of the flow could be modified independently of the length scale of the flow.

Regardless, it is clear that the dominant features, namely a decrease in primary frequency, followed by synchronization to a period doubled state, are the same for all values of $R e$ tested. The similarity of this behavior to that from low-Re two-dimensional simulations suggests that the dynamics of the wake are still governed by structures that are essentially two-dimensional, even at higher $R e$. A number of studies exist that indicate forcing can increase the correlation in the spanwise direction. Poncet ${ }^{6}$ and Thiria, Goujon-Durand, and Wesfried ${ }^{7}$ both show that by rotationally oscillating the cylinder near the Strouhal frequency (the vortex shedding frequency from the unperturbed cylinder), spanwise correlation in the wake can be significantly increased. Leontini, Thompson, and Hourigan ${ }^{30}$ also showed that transverse oscillations near the Strouhal frequency could delay the onset of three-dimensional structures. A significant difference between rotational or transverse oscillation, and the streamwise oscillation of this study, is the symmetry of the forcing compared to that of the vortex street. The vortex street is spatio-temporally symmetric; evolution forward by half a period plus a reflection about the wake centreline gives the same solution. Rotational and transverse forcing share this symmetry. However, streamwise oscillation does not. Despite this, it might be expected that the forcing leads to an increase in spanwise correlation, and this might go some way to explain the similarity of the behavior at these higher $R e$ to the behavior in two-dimensional simulations.

The data presented for the circular and square cylinder thus far are for driven oscillations at the natural shedding frequency, i.e., $f_{\mathrm{d}}=f_{\text {so }}$. An outstanding question from these studies is that of whether it is the driving frequency, or the unperturbed cylinder frequency, that controls the dynamics, or if there is any interaction between them. To investigate this, a series of experiments have been conducted at a frequency slightly detuned from the natural shedding frequency, for the circular cylinder, at $R e=5000$.

Forcing at a slightly detuned frequency across the tested range $\left(f_{\mathrm{d}}=f_{\text {so }} \pm 7.5 \%\right)$ exhibits the same overall trend in reducing shedding frequency until locking to a period doubled state at higher amplitude as shown in Figure 4. Figure 4(a) shows that subharmonic locking is to the driving frequency $f_{\mathrm{d}}$, causing the variation in locking frequency value. If these curves are normalized 

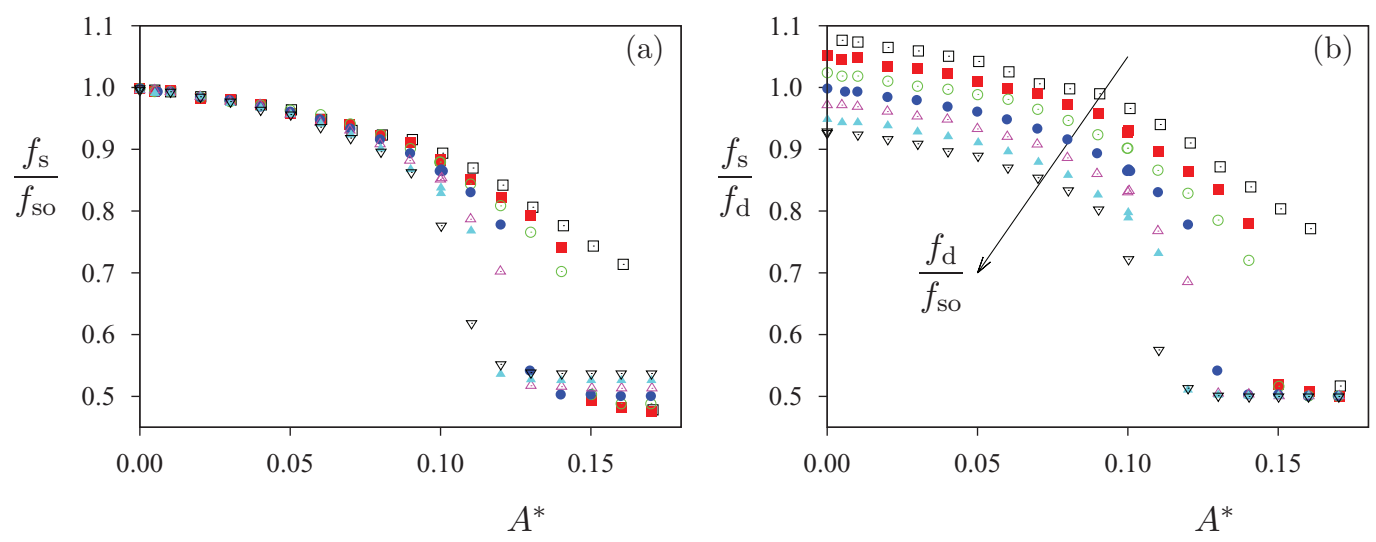

FIG. 4. The behaviour of $f_{\mathrm{s}}$ as a function of $A^{*}$, when the driving frequency $f_{\mathrm{d}}$ is detuned from the unperturbed shedding frequency $f_{\text {so }}$, for the circular cylinder at $R e=5000$, for values of $f_{\mathrm{d}} / f_{\mathrm{so}}: 0.925(\nabla), 0.950(\mathbf{\Delta}), 0.975(\triangle), 1.000(\bullet), 1.025$ (०), $1.050(\square), 1.075$ ( $\square$ ). (a) The primary shedding frequency, $f_{\mathrm{s}}$, normalized by $f_{\text {so }}$. (b) The primary shedding frequency, $f_{\mathrm{s}}$, normalized by $f_{\mathrm{d}}$. The plots clearly show that the shedding frequency varies smoothly with $A^{* 2}$ from the unperturbed cylinder case as $A^{*}$ is increased from $A^{*}=0$, and that the flow finally synchronizes to a subharmonic of the driving frequency, regardless of the driving frequency.

by forcing frequency instead of natural shedding frequency, as shown in Figure 4(b), the locking frequency collapses on to $f_{s} / f_{d}=0.5$. However, the forcing frequency has no effect at small amplitudes on the shedding frequency as shown in Figure 4(a). The shedding frequency is equal or close to that of the natural shedding.

For low amplitudes, the trend is still well approximated by a quadratic, regardless of the forcing frequency over the range tested. The coefficients of the power-law fits for the detuned shedding frequencies are presented in Table III. For all the values of the frequency tested, the exponent $b$ remains close to 2 .

In conclusion, this study has investigated the modes of response and trends in shedding frequency from a cylinder performing streamwise oscillations, where the frequency of this oscillation is equal, or close, to the vortex shedding frequency from an unperturbed cylinder.

For a circular cylinder, it has been shown that the initial trend of the primary frequency of shedding decreasing with $A^{* 2}$ holds at values of $R e \leqslant 6300$. The critical amplitude at which this trend ends with a sudden transition to a period doubled state has been shown to be a function of $R e$.

This initial trend of decreasing shedding frequency with $A^{* 2}$ also applies when the cylinder cross section is changed from circular to square. However, the transition to the period doubled state is not as abrupt with increasing $A^{*}$ for the square, as the primary frequency decreases smoothly to the subharmonic of the driving frequency over a small range of $A^{*}$. This may be due to competition between two modes of shedding.

At moderate amplitudes, it is the driving frequency, and not the unperturbed shedding frequency, that controls the dynamics. Experiments where the driving frequency for the circular cylinder is

TABLE III. Values of the coefficients of the power-law fit $f_{\mathrm{s}} / f_{\mathrm{d}}=c-a A^{* b}$, for the circular cylinder at $R e=5000$ as a function of the driving frequency $f_{\mathrm{d}}$. The exponent $b$ constantly remains close to 2 .

\begin{tabular}{lccr}
\hline \hline$f_{d} / f_{\text {so }}$ & $a$ & $b$ & $c$ \\
\hline 0.925 & 10.9 & 2.03 & 0.991 \\
0.950 & 14.4 & 2.11 & 0.992 \\
0.975 & 11.8 & 2.02 & 0.993 \\
1.000 & 16.2 & 2.10 & 0.993 \\
1.025 & 14.8 & 2.03 & 0.993 \\
1.050 & 28.2 & 2.25 & 0.990 \\
1.075 & 12.8 & 1.91 & 0.995 \\
\hline \hline
\end{tabular}


detuned from the unperturbed shedding frequency show the same trends as the case where the driving frequency is equal to the unperturbed shedding frequency, although the critical amplitude for the transition to the period doubled state, and the slope of the primary frequency of shedding prior to this transition, are functions of the driving frequency.

The authors would like to acknowledge the financial support of the Australian Research Council through Grant Nos. DP0774525 and DP110102141 as part of the discovery projects scheme, and the Australian Postdoctoral Fellowship of J.L.

${ }^{1}$ R. E. D. Bishop and A. Y. Hassan, "The lift and drag forces on a circular cylinder oscillating in a flowing fluid,” Proc. R. Soc. London, Ser. A 277, 51-75 (1964).

${ }^{2}$ C. H. K. Williamson and A. Roshko, "Vortex formation in the wake of an oscillating cylinder," J. Fluids Struct. 2, 355-381 (1988).

${ }^{3}$ H. M. Blackburn and R. Henderson, "A study of two-dimensional flow past an oscillating cylinder," J. Fluid Mech. 385, 255-286 (1999)

${ }^{4}$ J. S. Leontini, B. E. Stewart, M. C. Thompson, and K. Hourigan, "Wake-state and energy transitions of an oscillating cylinder at low Reynolds number," Phys. Fluids 18(6), 067101 (2006).

${ }^{5}$ J.-W. He, R. Glowinski, R. Metcalfe, A. Nordlander, and J. Periaux, "Active control and drag optimization for flow past a circular cylinder," J. Comput. Phys. 163, 83-117 (2000).

${ }^{6}$ P. Poncet, "Topological aspects of three-dimensional wakes behind rotary oscillating cylinders," J. Fluid Mech. 517, 27-53 (2004).

${ }^{7}$ B. Thiria, S. Goujon-Durand, and J. E. Wesfried, “The wake of a cylinder performing rotary oscillations,” J. Fluid Mech. 560, 123-147 (2006).

${ }^{8}$ J. D'Adamo, R. Godoy-Diana, and J. E. Wesfried, “Spatiotemporal spectral analysis of a forced cylinder wake,” Phys. Rev. E 84, 056308 (2011).

${ }^{9}$ H. M. Badr, M. Coutanceau, S. C. R. Dennis, and C. Ménard, "Unsteady flow past a rotating circular cylinder at Reynolds numbers $10^{3}$ and 104," J. Fluid Mech. 220, 459-484 (1990).

${ }^{10}$ S. Mittal and B. Kumar, "Flow past a rotating cylinder," J. Fluid Mech. 476, 303-334 (2003).

${ }^{11}$ P. A. Monkewitz and L. N. Nguyen, "Absolute instability in the near-wake of two-dimensional bluff bodies," J. Fluids Struct. 1, 165-184 (1987).

${ }^{12}$ G. E. Karniadakis and G. S. Triantafyllou, "Frequency selection and asymptotic states in laminar wakes," J. Fluid Mech. 199, 441-469 (1989).

${ }^{13}$ L. H. Feng and J. J. Wang, "Circular cylinder vortex-synchronization control with a synthetic jet positioned at the rear stagnation point," J. Fluid Mech. 662, 232-259 (2010).

${ }^{14}$ O. M. Griffin and S. E. Ramberg, "Vortex shedding from a cylinder vibrating in line with an incident uniform flow," J. Fluid Mech. 75, 257-271 (1976).

${ }^{15}$ C. Barbi, D. P. Favier, C. A. Maresca, and D. P. Telionis, "Vortex shedding and lock-on of a circular cylinder in oscillatory flow," J. Fluid Mech. 170, 527-544 (1986).

${ }^{16}$ E. Konstantinidis, S. Balabani, and M. Yianneskis, "The timing of vortex shedding in a cylinder wake imposed by periodic inflow perturbations," J. Fluid Mech. 543, 45-55 (2005).

${ }^{17}$ E. Konstantinidis and S. Balabani, "Symmetric vortex shedding in the near wake of a circular cylinder due to streamwise perturbations," J. Fluids Struct. 23, 1047-1063 (2007).

18 A. Ongoren and D. Rockwell, "Flow structure from an oscillating cylinder Part 2. Mode competition in the near wake," J. Fluid Mech. 191, 225 (1988).

${ }^{19}$ Y. Yokoi and K. Kamemoto, "Vortex shedding from an oscillating circular cylinder in a uniform flow," Exp. Therm. Fluid Sci. 8, 121-127 (1994).

${ }^{20}$ O. Cetiner and D. Rockwell, "Streamwise oscillations of a cylinder in a steady current. Part 1. Locked-on states of vortex formation and loading," J. Fluid Mech. 427, 1-28 (2001).

${ }^{21}$ P. G. Perdikaris, L. Kaitsis, and G. S. Triantafyllou, "Chaos in a cylinder wake due to forcing at the Strouhal frequency," Phys. Fluids 21, 101705 (2009).

22 J. S. Leontini, D. Lo Jacono, and M. C. Thompson, "A numerical study of an inline oscillating cylinder in a free stream," J. Fluid Mech. 688, 551-568 (2011).

${ }^{23}$ M. Nazarinia, D. Lo Jacono, M. C. Thompson, and J. Sheridan, "Flow behind a cylinder forced by a combination of oscillatory translational and rotational motions," Phys. Fluids 21, 051701 (2009).

${ }^{24}$ M. Sherry, D. Lo Jacono, and J. Sheridan, "An experimental investigation of the recirculation zone formed downstream of a forward facing step,” J. Wind. Eng. Ind. Aerodyn. 98, 888-894 (2010).

${ }^{25}$ M. Nazarinia, D. Lo Jacono, M. C. Thompson, and J. Sheridan, "The three-dimensional wake of a cylinder undergoing a combination of translational and rotational oscillation in a quiescent fluid," Phys. Fluids 21, 064101 (2009).

${ }^{26}$ M. S. Bloor, "The transition to turbulence in the wake of a circular cylinder," J. Fluid Mech. 19, 290-304 (1964).

${ }^{27}$ A. Roshko, "Perspectives on bluff body aerodynamics," J. Wind. Eng. Ind. Aerodyn. 49, 79-100 (1993).

${ }^{28}$ V. Parezanović and O. Cadot, "The impact of a local perturbation on global properties of a turbulent wake," Phys. Fluids 21, 071701 (2009).

${ }^{29}$ V. Parezanović and O. Cadot, "Experimental sensitivity analysis of the global properties of a two-dimensional turbulent wake,” J. Fluid Mech. 693, 115-149 (2012).

${ }^{30}$ J. S. Leontini, M. C. Thompson, and K. Hourigan, "Three-dimensional transition in the wake of a transversely oscillating cylinder," J. Fluid Mech. 577, 79-104 (2007). 